\section{Personal reinforcement and awareness}

\author{
WILLARD E. REITZ \\ University of Western Ontario, London, Ont., Canada
}

Ss were administered a visual discrimination task for which reinforcements were $A \cdot V-L$ value statements. Positive and negative rein forcers were chosen to be high in valency $( \pm 2 \mathrm{SD})$ or low $( \pm 1 \mathrm{SD})$. Awareness of reinforcement contingency was greater $(p<.001)$ under high-valency conditions than under low. The high-valency condition also resulted in higher performance and gains over trials. Results were interpreted as favoring a personal motive interpretation.

The concept of motivation is often used to cover all behavior. The belief, for example, that all behavior is motivated is a well-known postulate of drive-reduction theory. Peters (1958), however, feels that the construct of motivation is best used to explain only behavior which involves a personal goal and associated instrumental acts designed to achieve that goal. Other theorists have also made a similar point (e.g., McClelland, 1951; Maddi, 1968). In such conceptualizations, a distinction is made between drives and motives; drives are seen as being more biological and mechanical, as well as less self-conscious and intellective than motives. To reach the status of motive, a goal must be idiosyncratic to the person and achieve conscious mental representation. Both Maddi and McClelland push the matter further and also insist on distinguishing between motives of a society (schemas or social desirability) and that of the person. Recognizing the fact that a person may work toward goals of which he is unaware, Maddi urges a further elaboration to cover such contingencies: A personal goal need only have been conscious at one time (Maddi, 1968). In general, however, a motive is a personal goal that has achieved conscious mental representation.

Awareness thus plays a central role in this conceptualization. In studies of verbal reinforcement, it has similarly played an important role as an alternative cognitive explanatory concept to that of reinforcement. One view of awareness is that of an important cognitive mechanism which itself is underlain by reinforcement principles (Reitz \& McDougall, 1969). In this view, awareness emerges maximally under conditions where valued personal incentives are available. In the present study, the aim was to assess the hypothesis that awareness emerges as a function of intensity of personal motives. In general, it was expected that awareness would be stronger where highly valued reinforcements were available (high personal motives or valency), as contrasted with lesser valued ones (low valency). In addition, it was expected that performance would be greater under highly valued reinforcing conditions since, once emerged, awareness would be utilized to procure valued reinforcements.

SUBJECTS

A group of 90 nurses-in-training were administered the published version of the Allport-Vernon-Lindzey scale of values. From this initial pool, 25 Ss who met the criteria of the high-valency condition and 25 who met the criteria of the low-valency condition were selected. Criteria for high valency were one outstandingly high and one outstandingly low score (two standard deviations from the published mean in either direction) Low valency was defined as scores which were as close as possible to the published mean and not exceeding one standard deviation.

\section{PROCEDURE}

The learning task was a simple visual discrimination problem, as described by Golightly \& Byrne (1964). Briefly, Ss a wooden frame containing a cardboard center, with a window through which stimulus cards were presented. The window was in the lower left-hand corner from the S's view, thus ruling out eye contact throughout the experiment. On each of the 80 trials, a card containing a circle and a square appeared in which one object was black and one was white, one large and one small, and one on the left and one on the right. The large-small dimension was arbitrarily selected as the discrimination to be learned, with large correct for half the Ss and small correct for the other half. $S$ was told that this was a learning experiment in which she was to select one of two objects in each trial and to state her choice aloud. Immediately thereafter, a card was presented containing a single value statement through the same window opening which the $\mathrm{S}$ was to read aloud. In the high-valency condition, a correct response was followed by a value statement from the S's high value area, whereas an incorrect response was followed by a statement from the S's low value area. Ss read the statement aloud and then proceeded to the next trial. These value items were rewritten from the published test to be single sat in front of an apparatus consisting of statements espousing a given value. Exactly the same procedure was followed for the Ss in the low-valency group, except that the value statements were from an area in which $S$ had scored just above (for positive reinforcement) or below (for negative) the mean. Trials were continued for 80 replications or until there were eight successive correct (or incorrect) responses.

Following the learning aspect of the study, Ss were presented an awareness questionnaire to fill out. This questionnaire consisted of a series of statements regarding the reinforcement contingency, one of which was correct for each $S$. $S$ was asked to place a 1 beside the statement she felt took place during the study. If she was unsure of her choice, she should then place a 2 beside the statement of what she thought took place and a 3 beside another item if she was not sure of her second alternative.

\section{RESULTS}

Awareness results were scored by giving each $\mathrm{S}$ a score from 1 to $7 . \mathrm{A} 7,6$, or 5 was scored when $S$ gave the correct response contingency a rank of 1,2 , or 3 , respectively. A 4,3 , or 2 was scored when a rank of 1,2 , or 3 was given to an inappropriate statement but one which reflected the appropriate dimension-size. Finally, a 1 was scored if none of the responses were accurate.

A $t$ test was computed on the awareness scores which showed the high-valency group to be much more aware than the low-valency group $(t=3.35, \mathrm{df}=48, \mathrm{p}<.001)$. Thus, as predicted, awareness was higher under conditions where incentives available were highly valued (high mean $=4.48$; low mean $=2.04$ ). In addition, Ss reaching a positive criterion in the high-valency condition $(n=13)$ were significantly higher in awareness than those reaching the same criterion in the low condition $(n=7, t=2.32, d f=18$, $\mathrm{p}<.05$ ). Awareness was, however, unrelated to how soon Ss reached criterion in either direction. This lack of relationship held true for the high-and low-valency conditions singly and in combination. In addition, there was no relationship found between awareness and performance in either experimental condition, irrespective of whether Ss reached criterion.

Performance results are depicted graphically in Fig. 1. A 2 by 5 analysis of variance computed on the performance data revealed a significant effect for trials $(\mathrm{F}=4.11, \mathrm{df}=1 / 48$, $\mathrm{p}<.05$ ), indicating an increase of objects chosen over trials which brought forth value statements in line with the S's own values. In addition, there was a significant effect of groups $(\mathrm{F}=2.72, \quad \mathrm{df}=4 / 192, \quad \mathrm{p}<.05)$, indicating that $\mathrm{Ss}$ in the high-valency condition performed better in the sense 
of choosing objects which brought forth value statements from their highly valued area. The lack of a Group by Trials interaction is due to an increase in variance as trials proceeded, especially in the high-valency condition. This result, in turn, is due to the scoring procedure where, once reaching criterion, maximal (16) or minimal (0) scores were automatically assigned throughout the remaining blocks. Three people in the high-valency condition performed to criterion in the nonpredicted direction, and 13 performed to criterion in the predicted direction. These results brought about a condition of increasing variance with trials in the high-valency condition. In the low-valency condition, however, five $S s$ reached criterion in the nonpredicted direction and seven in the predicted direction, resulting in less variance.

Most Ss obtained their highest score in the social-value area $(86 \%$ and nondifferentially in the two groups). None obtained their lowest score in the social area but all other areas were represented as low. Classifying only on the lows, no tendency was found for any given area to either be more effective reinforcers or be related to awareness ( Fs $<1.0$ ).

\section{DISCUSSION}

Results are consistent with numerous past studies in demonstrating that attitudinal stimuli can be substituted for more traditional reinforcements and bring about response alteration. Furthermore, response alteration is mediated by awareness, but awareness is maximized under conditions where the reinforcements are personally valued by the $S$ and appropriately contingent. Since each $S$ received reinforcing statements from only two value domains, heterogeneity of domain must be ruled out as a determining variable, at least under the conditions studied. This result is consistent with earlier results of Reitz et al (1968), where personal valency was interpreted as being a central variable in affecting the reinforcement property of attitude statements. The result, however, is at variance with Byrne et al's (1968) conclusion that homogeneity alone determines the reinforcing value of attitudinal stimuli. The Byrne et al findings, however, are confounded with the differential number of reinforcing items used. That is, their homogeneous condition consisted of two statements repeated over and over, whereas in the heterogeneous condition, 90 items were used. This variable was controlled in the present study by using an equal number of reinforcing items for each $S$, regardless of experimental condition. Homogeneity has been shown to be an important factor as well (Reitz et al,

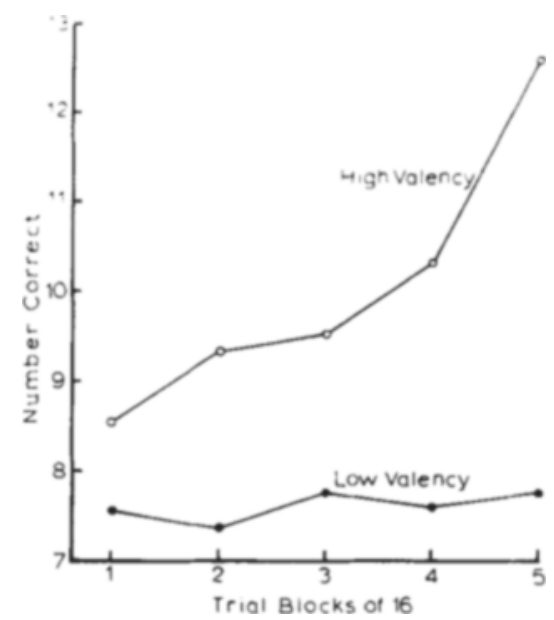

Fig. 1. Performance curves of the highand low-valency conditions.

1968), but personal valency, centrality, or magnitude of reinforcement is clearly implicated in the present results.

The results contribute to the cognitive vs reinforcement controversy in verbal learning by specifying conditions under which awareness is most likely to develop. While a wareness may, under some conditions, emerge in the (relative) absence of personal incentives (Reitz \& McDougall, 1969), it is not thus maximized and is less likely to affect or mediate performance. When valued personal incentives are available, however, awareness of the reinforcement contingency emerges, and $S$ performs in such a way as to obtain the incentives. Thus, a view of awareness is suggested in which awareness is an important cognitive mechanism which makes possible the maximization of personal goals. From an evolutionary. vantage, the development of awareness was facilitated by the natural selection of those species which could maximally satisfy their needs. At the individual level, awareness becomes a vehicle for maximizing the reinforcement potential of environmental stimuli which are of personal interest. This view of awareness is consistent with Collier's (1956) conception of awareness as a regulatory field and Sperry's (1969) view of conscious awareness as an emergent quality of brain activity.

A puristic cognitive theorist might insist that the Ss in the present study were responding only to the informational aspects of the reinforcing stimuli. That is, Ss might be expected to assume the task to be of a correct-incorrect nature, with the reinforcing items serving only as cues. Such a position leaves unexplained why Ss "assume" their highly valued domain to be "correct" and their lowly valued domain "incorrect." There is nothing in the instructions to indicate which domain might be correct or incorrect. Furthermore, even if they did assume their highly valued domain was correct as the basis of their performance, the fact that more of them make this assumption (judging from the number of Ss reaching either criterion), as opposed to assuming that their highly valued domain is incorrect, is itself significant ( 3 vs $13, \mathrm{p}=.01$, sign test). In addition, continuing in this framework, slightly more $\mathrm{Ss}$ in the low-valency condition than in the high make the assumption that their higher valued area is incorrect and fewer assume it to be correct ( 5 vs $7, p=.39$, sign test). These results are thus inconsistent with the puristic cognitive or a demand characteristics explanation as determining Ss' performance.

Those Ss who went in the nonpredicted direction pose an interesting problem. While there were few of them in this particular study, making post hoc internal analyses unreliable, in past studies (e.g., Reitz, 1969) awareness analyses became meaningful only when separating out such groups. Various interpretations are possible. Such Ss, for example, may be more tolerant of other lowly valued areas or they may be brighter, seeing through the contingency quickly and wanting to seek out other less familiar values. These in terpretations and others that could be made require further comparative evaluation.

The quasiexperimental manipulation of personal characteristics (in this case, values) also blunts the critics of Skinner who feel the reinforcement principle to be nonpredictive at the human level (cf. Carcini, 1969). That is, the operation of food (or water, sex, etc.) deprivation in animals makes prediction of reinforcers possible. At the human level, it is claimed that reinforcers can only be known after the fact. Studies employing reliable personality measurement, however, can be expected to reasonably predict what will be reinforcing and thus, at least in part, reduce this criticism. In addition, awareness itself can be seen as an operant which is underlain by and subject to principles of reinforcement.

\section{REFERENCES}

BYRNE, D., GRIFFITT, W., \& CLORE, G. L Attitudinal reinforcement effects as a f un t i o n of st i m u l u s homogeneity-heterogeneity. Journal of Verbal Learning \& Verbal Behavior, 1968. 7.962-964

CARINI, L. The fault in Skinner's teaching machine. American Psychologist, 1969, 24, 471-473.

COLLIER, R. W. Consciousness as a regulatory field: A theory of psychopathology. Psychological Review, $1956,63,360-369$.

GOLIGHTLY, C., \& BYRNE, D. Attitude statements as positive and negative reinforcements. Science, 1964, 146, 798-799. 
1] ADDI, S. fersomalily heories: $A$ comparatieg analysis. Nobleton, Ontario: Irwin-Dorsey Press, 1968.

McClELLAND, D. C. Fersonatity. New York: Dryden, 1951.

PETERS, R. S. The concepl of molivation. New York: Humanities Press, 1958

REITZ, $W$, E., DOUEY, J. \& MASON, G. Role of homogeneity and centrality of at titude domain on reinforcing properties of attitude statements. Journal of Experimental Restarch in Personality. $1968,3,120-125$.

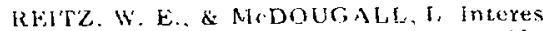
ilems as positive and negative reinforcements: Effects of social desirability and extremity of endorsement. Psychonomic Science. 1969, 17, 97-98.

REITZ, W. E. Effect of homogeneity, valency and awareness on attitudinal reinforcement and interpersonal attraction. Research Bulletin No.118, 1969, University of Westerm Ontario, London, Canada.

SPERRY, R. W. A modified concept of consciousness. Psychological Review, $1969,76,532-536$. statements received from the stranger (cf. Byrne, 1969). In the studies in which this relationship was found, the order in which similar and dissimilar attitude statements were received from the stranger were randomized, with the restriction of maintaining specific proportions of similar statements for particular experimental conditions. Recently, Byrne and his associates have begun to explore the effects on attraction toward a stranger of the sequences in which the similar and dissimilar attitude statements were received.

Byrne \& London (1966) held overall proportion of similar attitude statements constant and compared the effects of two sequences. In a similar-dissimilar sequence, the following numbers of similar and dissimilar attitude statements were presented within blocks of eight statements: $8-0,7-1,6-2,4-4,2-6,1-7$, $0-8$. For the dissimilar-similar sequence, this pattern was reversed. The two sequences did not result in any difference in attraction measured at the end of the entire sequence.

Byrne, Lamberth, Palmer, \& London (1969) varied the sequence of presentation and measured attraction after each new attitude statement was presented. They found that S's attraction to this stranger was a function of the most recent attitude statements rather than a function of the overall proportion of similar attitude statements. They concluded that "sequential effects occur in the attitude-attraction relationship when subjects make evaluative responses during the sequence [p. 76].'”

An examination of the item-by-item responses collected by Byrne et al (1969) revealed that large differences in attraction responses were made when there were shifts from similar to dissimilar attitude statements or vice versa, while strength of response was relatively stable across items when no shift occurred. From further examination of item-by-item responses, it appears that with repeated presentation of similar attitude statements, there is no incremental growth in the strength of attraction. Apparently, attraction is a well-learned response which is evoked immediately in full strength at the first presentation of a similar attitude statement. Similar and dissimilar attitude statements may serve as discriminative stimuli, which control discrete responses of different strengths based on experience prior to the experiment. This would imply that, with a continuous mode of responding, previous exposure to attitude statements would have no effect on responses following later

attitude statements.
* Based on an honor's thesis submitted to Syracuse University by the first author under the direction of the second author.

+Now at Johns Hopkins University, Baltimore, Md. 21218 .
Byrne and his associates have presented consistent evidence that a S's attraction toward a stranger is a positive linear function of the proportion of similar attitude 